\section{CONHECIMENTO E PRÁTICA DE MÉDICOS E ENFERMEIROS SOBRE DETECÇÃO PRECOCE DO CÂNCER DE MAMA}

\author{
Knowledge and practice of doctors and nurses regarding early \\ detection of breast cancer
}
Conocimiento y práctica de médicos y enfermeros sobre detección precoz del cáncer de mama

\section{RESUMO}

Objetivo: Comparar o conhecimento e a prática autorreferida dos médicos e enfermeiros atuantes na Estratégia de Saúde da Família em relação à detecção precoce do câncer de mama. Métodos: Estudo transversal realizado no município de Campo Grande, Mato Grosso do Sul, com médicos e enfermeiros atuantes na Estratégia Saúde da Família, entre 2011 e 2012. Utilizou-se um questionário estruturado para investigação das variáveis sociodemográficas e conhecimento e prática no que diz respeito à detecção precoce do câncer de mama. A avaliação da associação entre a categoria profissional e as demais variáveis foi realizada por meio do teste Qui-quadrado, considerando nível de significância de 5\%. Resultados: Foram entrevistados 109 profissionais, dos quais 49,5\% ( $n=54)$ eram médicos e $50,5 \%(n=55)$ eram enfermeiros. Destes, $72,2 \%(n=39)$ dos médicos e $87,2 \%(n=48)$ dos enfermeiros haviam recebido capacitações referentes ao câncer de mama. Quanto ao exame mais indicado para a detecção precoce do câncer de mama, houve diferença significativa para mamografia $(\mathrm{p}<0,001)$, sendo indicada por $50 \%(\mathrm{n}=50)$ dos médicos e $13,2 \%(\mathrm{n}=7)$ dos enfermeiros. Conclusão: Houve diferenças significativas tanto no conhecimento quanto na prática referentes às ações para a detecção precoce do $\mathrm{CM}$ entre médicos e enfermeiros atuantes na ESF. Os médicos apresentaram maior conhecimento sobre a mamografia como método mais adequado para detecção em estágio iniciais do CM quando comparados aos enfermeiros. No que concerne à prática, o ECM é realizado abaixo do esperado, tanto por médicos como por enfermeiros.

Descritores: Neoplasias da Mama; Estratégia Saúde da Família; Prática Profissional.

\section{ABSTRACT}

Objective: To compare the self-reported knowledge and practice of doctors and nurses of the Family Health Strategy regarding early detection of breast cancer. Methods: Cross-sectional study conducted in the municipality of Campo Grande, Mato Grosso do Sul, with doctors and nurses of the Family Health Strategy between 2011 and 2012. A structured questionnaire was used to assess sociodemographic variables and knowledge and practice regarding early detection of breast cancer. The assessment of the association between the professional class and the other variables was performed using the Chi-squared test with a significance level of 5\%. Results: 109 professionals were interviewed: $49.5 \%(n=54)$ were doctors and $50.5 \%$ $(n=55)$ were nurses. Of these, $72.2 \%(n=39)$ of the doctors and $89.1 \%(n=48)$ of the nurses had received training on breast cancer. As for the most indicated exam for early detection of breast cancer, there was significant difference regarding mammography $(p<0.001)$, which was indicated by 50\% $(n=50)$ of the doctors and $13.2 \%(n=7)$ of the nurses. Conclusion. There were significant differences both in the knowledge and in the practice regarding the actions for early detection of breast cancer between doctors and nurses working in the FHS. Doctors presented greater knowledge of mammography as the most adequate method for the detection of $B C$ in its earliest stages when compared to nurses. Regarding the practice, the $C B E$ is performed less than expected by both doctors and nurses.

Descriptors: Breast Neoplasms; Family Health Strategy; Professional Practice.
Artigo Original
1) Universidade Federal de Mato Grosso do Sul - UFMS - Campo Grande (MS) - Brasil
Recebido em: 07/10/2015 Revisado em: 02/03/2016 Aceito em: 28/04/2016 


\section{RESUMEN}

Objetivo: Comparar el conocimiento y la práctica auto referida de médicos y enfermeros de la Estrategia de Salud de la Familia respecto la detección precoz del cáncer de mama. Métodos: Estudio transversal realizado en el municipio de Campo Grande, Mato Grosso do Sul, con médicos y enfermeros de la Estrategia de Salud de la Familia entre 2011 y 2012. Se utilizó un cuestionario estructurado para investigar las variables sociodemográficas, el conocimiento y la práctica respecto la detección precoz del cáncer de mama. La valoración de la asociación entre la categoría profesional y las demás variables se dio a través de la prueba Chi-cuadrado, considerando el nivel de significación del 5\%. Resultados: Fueron entrevistados 109 profesionales de los cuales el 49,5\% $(n=54)$ eran médicos y el 50,5\% $(n=55)$ enfermeros. De estos, el 72,2\% $(n=39)$ de los médicos y el 87,2\% $(n=48)$ de los enfermeros recibieron capacitaciones sobre el cáncer de mama. Respecto la prueba más indicada para la detección precoz del cáncer de mama, hubo diferencia significativa para la mamografía $(p<0,001)$ que fue indicada por el 50\% $(n=50)$ de los médicos $y$ el 13,2\% $(n=7)$ de los enfermeros. Conclusión: Hubo diferencias significativas para el conocimiento y la práctica de las acciones de detección precoz del CM entre los médicos y los enfermeros de la ESF. Los médicos presentaron más conocimiento sobre la mamografía como método más adecuado para la detección en fase inicial del CM al comparar con los enfermeros. Respecto la práctica, el ECM es menos realizado de los que se espera de los médicos y enfermeros.

Descriptores: Neoplasias de la mama; Estrategia de Salud Familiar; Práctica Profesional.

\section{INTRODUÇÃO}

Entre as neoplasias malignas mais comuns, o câncer de mama (CM) é a que mais acomete as mulheres no mundo. Segundo as estimativas do Instituto Nacional de Câncer (INCA) para o ano de 2016, são esperados 57.960 novos casos de CM no país, o que representa 56,20 casos a cada 100 mil mulheres ${ }^{(1)}$. Para o estado do Mato Grosso do Sul, estima-se que 820 mulheres receberão o diagnóstico de $\mathrm{CM}$ nesse mesmo ano, sendo 460 na capital, Campo Grande ${ }^{(1)}$.

O CM apresenta-se como uma doença de máxima importância para a saúde pública no país devido à sua elevada incidência. As medidas de prevenção primária são bastante promissoras quando se considera que cerca de $30 \%$ dos casos de $\mathrm{CM}$ poderiam ser evitados por hábitos e comportamentos saudáveis ${ }^{(1)}$. Entretanto, esse tipo de prevenção não é totalmente possível devido à multicausalidade envolvida em sua etiologia, não sendo possível prever o surgimento da doença $\mathrm{a}^{(2,3)}$.

As estratégias de prevenção secundária do CM devem basear-se na detecção precoce da doença por meio de medidas, como o diagnóstico precoce, em que a abordagem se faz em indivíduos com sinais e/ou sintomas da doença, e do rastreamento, que se dirige à população assintomática com a realização de testes ou exames ${ }^{(4)}$, como a mamografia e o exame clínico das mamas $(\mathrm{ECM})^{(5)}$.

A detecção precoce baseia-se no conceito de que quanto mais cedo o câncer é detectado e diagnosticado, melhor será o prognóstico, aumentando a possibilidade de cura e sobrevida das mulheres ${ }^{(5-7)}$. As elevadas taxas de morbimortalidade no país associadas a esse tipo de câncer provavelmente se devem ao diagnóstico tardio. Recomendase a associação das práticas de prevenção primária e secundária ${ }^{(8)}$, visando à redução de tais índices e criação de políticas voltadas à saúde da mulher, que possam garantir a elas acesso a ações efetivas de rastreamento e diagnóstico precoce $^{(9)}$.

As ações quanto ao $\mathrm{CM}$ no Brasil devem ser coordenadas pela Atenção Primária à Saúde (APS), que tem como centralidade a Estratégia de Saúde da Família (ESF) ${ }^{(10,11)}$. Esse nível de atenção está próximo ao usuário, com função de acompanhá-lo longitudinalmente, constituindo-se porta de entrada preferencial para o Sistema Único de Saúde (SUS), oferecendo serviços de promoção, prevenção, cura e reabilitação para otimizar a saúde e o bem-estar dos indivíduos ${ }^{(12)}$. As estratégias para o enfrentamento do $\mathrm{CM}$ devem englobar a comunicação efetiva com a população, alertando-a para o aparecimento de sinais e sintomas, aumentando sua adesão às ações propostas, além da capacitação contínua dos profissionais, a fim de melhorar sua capacidade de diagnóstico e tomada de decisão, bem como a organização de serviços que garantam a integralidade da assistência durante todo o tratamento do $\mathrm{CM}^{(13)}$.

Os médicos e enfermeiros desempenham significativo papel na promoção da saúde, prevenção e diagnóstico do $\mathrm{CM}$ entre os profissionais que compõem a equipe da ESF, assim como no acompanhamento das mulheres acometidas. A solicitação do exame de mamografia está entre as atribuições especificas dos médicos para o controle de CM, tal como a de exames complementares se necessário, como a ultrassonografia. A identificação da população vulnerável, a realização do ECM, a condução de atividades de educação em saúde, a realização de cuidados paliativos, entre outros $^{(10)}$, encontram-se entre as atribuições de profissionais médicos e enfermeiros.

O aumento nas taxas de incidência, prevalência e mortalidade relativas ao $\mathrm{CM}$ indicam a necessidade contínua de reorganização de ações na APS no que concerne ao diagnóstico e tratamentos oportunos. A promoção da saúde, por meio de adoção de hábitos como alimentação adequada, controle do peso corporal, prática regular de exercícios e abandono de consumo de tabaco e álcool, torna-se preponderante para a melhoria dos indicadores 
da doença ${ }^{(10)}$. Dessa forma, é primordial a ampliação da comunicação entre profissionais, população e serviços de saúde para a disseminação de informações sobre fatores de risco, sinais, sintomas, medidas de promoção da saúde e prevenção da doença.

Isso posto, objetivou-se comparar o conhecimento e a prática autorreferida dos médicos e enfermeiros atuantes na ESF em relação à detecção precoce do $\mathrm{CM}$, tendo em vista a importância da atuação desses profissionais nas ações concernentes ao CM.

\section{MÉTODOS}

Trata-se de um estudo transversal realizado no município de Campo Grande, Mato Grosso do Sul, no período compreendido entre os meses de agosto de 2011 e agosto de 2012. Realizou-se o estudo em todas as 27 Unidades Básicas de Saúde da Família (UBSF) localizadas na área urbana de Campo Grande, totalizando 64 equipes de ESF.

A amostra inicial deste estudo partiu da listagem de todos os 128 profissionais de saúde, sendo 64 médicos e 64 enfermeiros em atividade na ESF, responsáveis pelas ações referentes à deteç̧ão precoce do CM. Entretanto, excluíram-se os profissionais que por algum motivo não se encontravam na UBSF ou se recusaram a participar do estudo, o que resultou em uma amostra final de 109 profissionais, sendo 54 médicos e 55 enfermeiros.

Utilizou-se um questionário estruturado previamente elaborado e de autopreenchimento para a realização da pesquisa. Os profissionais foram identificados por meio de contato com os gerentes das UBSFs, e em seguida contatados e convidados a participar do estudo, com posterior agendamento da visita das pesquisadoras para aplicação do instrumento de pesquisa.

Questões que buscaram avaliar variáveis sociodemográficas e conhecimento e prática quanto à deteç̧ão precoce do $\mathrm{CM}$ na APS compuseram o questionário, comparando os resultados obtidos entre os profissionais médicos e os enfermeiros.

Realizou-se a avaliação da associação entre a formação profissional dos indivíduos entrevistados e as demais variáveis avaliadas neste estudo por meio do teste Quiquadrado. Apresentaram-se os demais resultados das variáveis avaliadas na forma de estatística descritiva ou na forma de tabelas e gráficos. Realizou-se a análise estatística utilizando-se o software SPSS, versão 20.0, ou SigmaPlot, versão 12.5 , considerando um nível de significância de $5 \%{ }^{(14)}$.

Todos os participantes receberam esclarecimentos sobre os objetivos e sobre a metodologia da pesquisa. Os que aceitaram participar do estudo assinaram o termo de consentimento livre e esclarecido, atendendo-se à Resolução CNS 466/2012. O estudo foi aprovado pelo Comitê de Ética em Pesquisa da Universidade Federal de Mato Grosso do Sul, protocolo $\mathrm{n}^{\mathrm{o}} 1.719$.

\section{RESULTADOS}

Responderam ao questionário 109 profissionais, sendo $49,5 \%$ médicos e $50,5 \%$ enfermeiros. A idade dos médicos participantes variou de 23 a 60 anos de idade, sendo a idade média de $35,26 \pm 1,48$ anos (média \pm erro padrão da média). A idade dos enfermeiros variou de 25 a 59 anos, sendo a idade média de $36,31 \pm 1,28$ anos. Não houve diferença significativa entre médicos e enfermeiros no tocante à idade (teste $\mathrm{T}$-student, $\mathrm{p}=0$,598). A maioria da população compôs-se por profissionais do sexo feminino, tanto entre os médicos $(51,9 \%)$ quanto entre os enfermeiros $(89,1 \%)$ $(\mathrm{p}<0,001)$.

Entre os participantes, $72,2 \%$ dos médicos e $89,1 \%$ dos enfermeiros haviam recebido capacitação específica para a realização de ações relativas ao $\mathrm{CM}$. Entre os médicos e enfermeiros que receberam capacitação, $24,1 \%$ e $36,4 \%$, respectivamente, apontaram que esta foi insuficiente $(\mathrm{p}=0,064)$.

A opinião dos participantes divergiu quanto ao exame mais indicado para a detecção precoce do CM. Observou-se que $28 \%$ dos médicos e $45,3 \%$ dos enfermeiros citaram o Autoexame das mamas (AEM), enquanto $22 \%$ dos médicos e $41,5 \%$ dos enfermeiros citaram o ECM. A mamografia foi considerada como o método mais indicado para a detecção precoce do CM por 50\% dos médicos e 13,2\% dos enfermeiros $(\mathrm{p}<0,001)$. Tais resultados estão demonstrados na Figura 1.

No que diz respeito à prática do ECM no cotidiano da ESF, a quase totalidade dos profissionais consideraram-se capacitados para a realização do exame. O ECM foi citado como um método seguro para a detecção precoce do $\mathrm{CM}$ por $58,5 \%$ dos médicos e $81,5 \%$ dos enfermeiros $(\mathrm{p}=0,017)$. Embora se sintam capacitados para a realização do ECM e o considerem um exame confiável para a detecção precoce do $\mathrm{CM}$, o exame é ofertado para as mulheres durante consultas clínicas de rotina por $61,1 \%$ dos médicos e $51,9 \%$ dos enfermeiros $(\mathrm{p}=0,438)$. Quando questionados em quais situações o ECM é priorizado e realizado, $90,9 \%$ dos enfermeiros e $35,2 \%$ dos médicos $(\mathrm{p}<0,001)$ citaram o momento da coleta do exame Papanicolau. Para 72,2\% dos médicos e $49,1 \%$ dos enfermeiros, o ECM é realizado em mulheres que apresentem sinais e sintomas, como nódulos, dor, descarga papilar e outros $(\mathrm{p}=0,013)$. Estão demonstrados os resultados sobre a prática dos profissionais no que se refere ao ECM na Tabela I. 


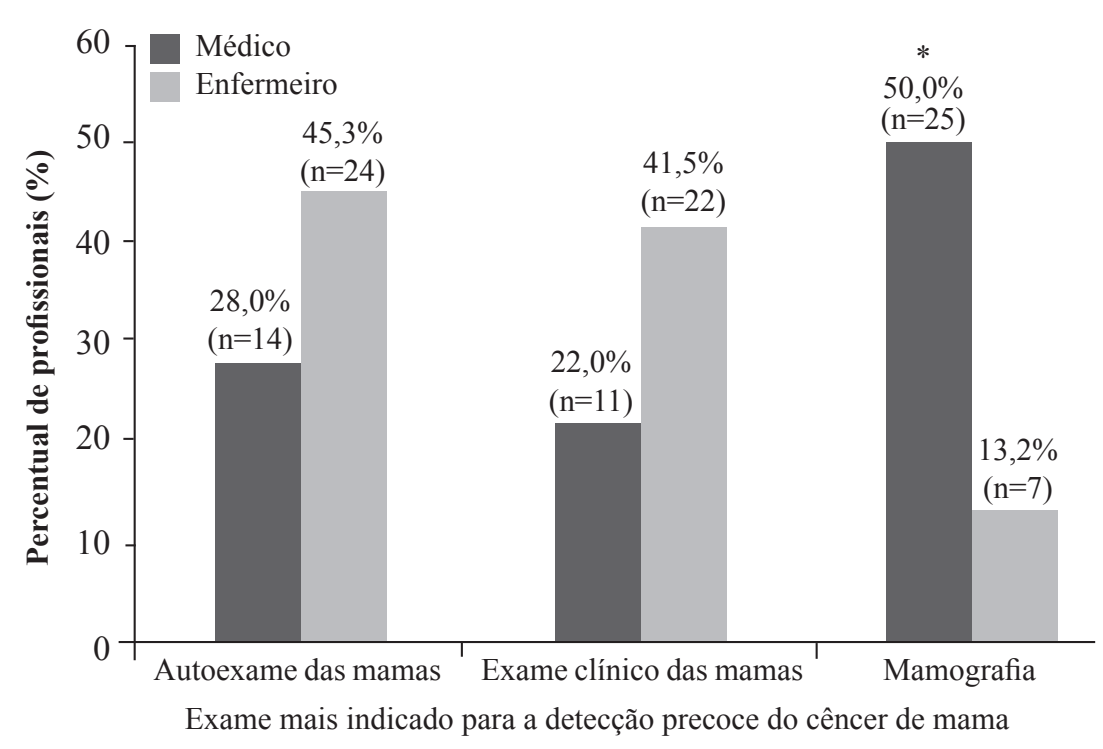

Figura 1 - Distribuições de profissionais de acordo com os tipos de exames mais apropriado para a detecção precoce do câncer de mama. Campo Grande, MS, 2012.

${ }^{*} \mathrm{p}<0,05$, Teste Qui-quadrado

Tabela I - Distribuição dos profissionais de acordo com as situações que prioriza/realiza o exame clínico de mama e a percepção sobre esse exame. Campo Grande, MS, 2012.

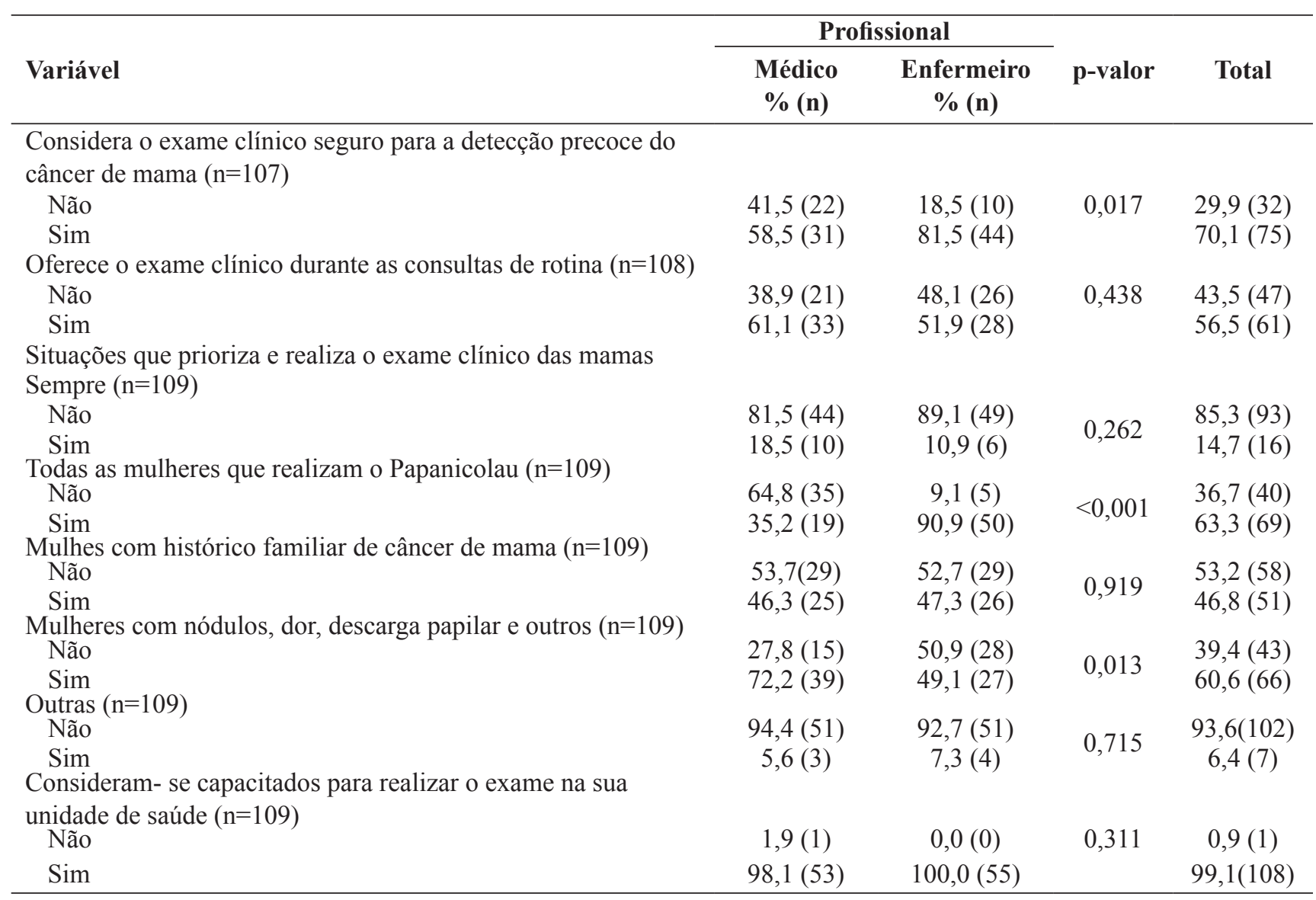




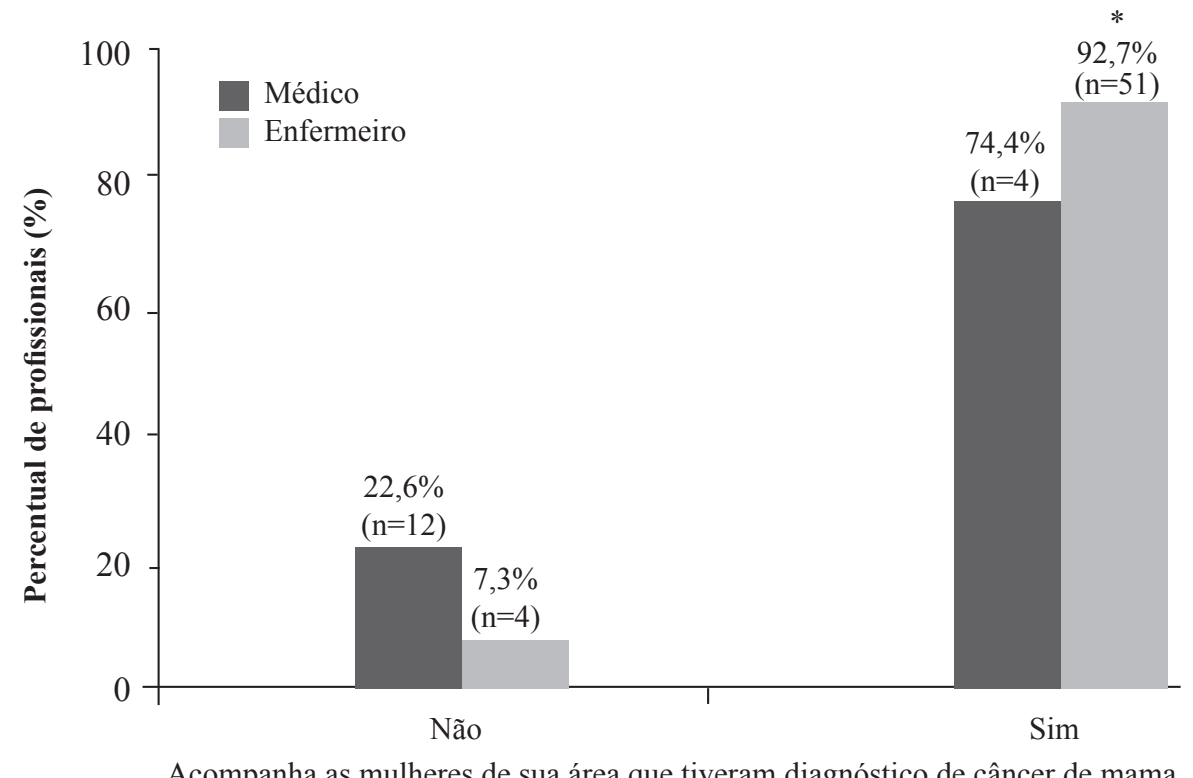

Figura 2 - Distribuições de profissionais em relação ao acompanhamento das mulheres de sua área que tiveram diagnóstico de câncer de mama. Campo Grande, MS, 2012.

${ }^{*} \mathrm{p}<0,05$, Teste Qui-quadrado.

Tabela II - Distribuição dos profissionais de acordo com os fatores que interferem na qualidade do exame clínico das mamas. Campo Grande-MS, 2012 (n=109).

\begin{tabular}{|c|c|c|c|c|}
\hline \multirow[b]{2}{*}{ Variável } & \multicolumn{2}{|c|}{ Profissional } & \multirow[b]{2}{*}{ p-valor } & \multirow[b]{2}{*}{ Total } \\
\hline & $\begin{array}{l}\text { Médico } \\
\% \text { (n) }\end{array}$ & $\begin{array}{c}\text { Enfermeiro } \\
\% \text { (n) }\end{array}$ & & \\
\hline \multicolumn{5}{|c|}{ Especialidade do profissional em Ginecologia e Obstetrícia } \\
\hline Não & $85,2(46)$ & $89,1(49)$ & \multirow{2}{*}{0,542} & $87,2(95)$ \\
\hline Sim & $14,8(8)$ & $10,9(6)$ & & $12,8(14)$ \\
\hline \multicolumn{5}{|c|}{ Tempo dedicado ao exame } \\
\hline Não & $42,6(23)$ & $52,7(29)$ & \multirow{2}{*}{0,290} & $47,7(52)$ \\
\hline Sim & $57,4(31)$ & $47,3(26)$ & & $52,3(57)$ \\
\hline \multicolumn{5}{|c|}{ Capacitações permanentes } \\
\hline Não & $59,3(32)$ & $50,9(28)$ & \multirow{2}{*}{0,381} & $55,0(60)$ \\
\hline Sim & $40,7(22)$ & $49,1(27)$ & & $45,0(49)$ \\
\hline \multicolumn{5}{|c|}{ Tempo de atuação na ESF } \\
\hline Não & $87,0(47)$ & $94,5(52)$ & \multirow{2}{*}{0,175} & $90,8(99)$ \\
\hline Sim & $13,0(7)$ & $5,5(3)$ & & $9,2(10)$ \\
\hline \multicolumn{5}{|c|}{ Frequência do exame clínico das mamas em suas pacientes } \\
\hline Não & $48,1(26)$ & $47,3(26)$ & \multirow{2}{*}{0,927} & $47,7(52)$ \\
\hline Sim & $51,9(28)$ & $52,7(29)$ & & $52,3(57)$ \\
\hline \multicolumn{5}{|l|}{ Outros } \\
\hline Não & $96,3(52)$ & $98,2(54)$ & \multirow{2}{*}{0,547} & $97,2(106)$ \\
\hline $\operatorname{Sim}$ & $3,7(2)$ & $1,8(1)$ & & $2,8(3)$ \\
\hline
\end{tabular}

ESF: Estratégia de Saúde da Família 
Não se observou diferença significativa entre as respostas dos profissionais médicos e enfermeiros $(\mathrm{p}>0,05)$ no que se refere aos fatores capazes de influenciar a qualidade do ECM, sendo o tempo que o profissional dedica ao exame e a frequência com que realiza o exame nas mulheres por eles atendidas os fatores mais reconhecidos, dados demonstrados na Tabela II.

Além das ações destinadas ao diagnóstico do $\mathrm{CM}$, investigou-se neste estudo a frequência com que médicos e enfermeiros da ESF acompanham as mulheres após o diagnóstico de $\mathrm{CM}$ durante e após o tratamento realizado em outros níveis de complexidade do sistema de saúde. Conforme a Figura 2, 92,7\% dos enfermeiros e 77,4\% dos médicos $(\mathrm{p}=0,048)$ relataram o acompanhamento das mulheres.

\section{DISCUSSÃO}

O cuidado em saúde desenvolvido na APS é fundamental para a prevenção e detecção precoce de doenças como o $\mathrm{CM}$, visto que está diretamente relacionado com a diminuição dos índices de mortalidade e agravos em saúde na população ${ }^{(15)}$.

No tocante à realização de capacitações para ações referentes ao $\mathrm{CM}$, grande parte dos médicos e enfermeiros incluídos neste estudo havia realizado capacitações especificas. Esse resultado representa um número muito superior ao encontrado no estudo realizado na cidade de Mossoró, Rio Grande do Norte, no qual apenas 12,1\% dos médicos e menos da metade dos enfermeiros $(44,7 \%)$ haviam recebido tais capacitações ${ }^{(16)}$. É importante elucidar que as ações de capacitação profissional devem ser realizadas de forma contínua, independentemente da frequência de novos casos da doença ${ }^{(17,18)}$. Apesar de a maioria dos profissionais estudados ter recebido capacitações, grande parte considerou-as insuficientes para o desenvolvimento de ações de detecção precoce do câncer de mama, o que pode refletir na prática reduzida dos profissionais para a realização de exames, como o ECM, por exemplo.

Em outro estudo, realizado com o objetivo de avaliar a prática do ECM por enfermeiros atuantes na ESF, demonstrou-se que a maioria dos participantes do estudo possuía conhecimento técnico e teórico para o desenvolvimento de ações que concernem à detecção precoce do $\mathrm{CM}$, sendo tal conhecimento considerado indispensável ao contexto da atenção primária para a realização eficaz da promoção da saúde e prevenção de agravos $^{(19)}$. Sugere-se que o grau de conhecimento do profissional - mas principalmente a sua segurança quanto à realização do ECM - pode estar relacionado tanto com a eficácia dele quanto com a oferta dessa ação no cotidiano dos serviços.
A detecção precoce do $\mathrm{CM}$ por intermédio da realização do exame mamográfico é indicada para mulheres com idade entre 50 e 69 anos, bianualmente, e por meio do ECM em todas as mulheres a partir dos 40 anos de idade, anualmente. As mulheres com risco elevado de desenvolver o $\mathrm{CM}$ devem realizar a mamografia e o ECM anualmente ${ }^{(10,20)}$. Tais recomendações devem ser de conhecimento dos profissionais médicos e enfermeiros atuantes na ESF, considerando seu papel decisivo no processo de rastreamento e deteç̧ão precoce.

É crescente o interesse pelas diferentes estratégias capazes de detectar o $\mathrm{CM}$ em estágios iniciais ${ }^{(3)}$, e vários estudos demonstram a associação da realização do rastreamento mamográfico populacional e a redução da mortalidade por $\mathrm{CM}^{(21-23)}$. Atualmente, a mamografia é considerada o exame mais eficaz para a detecção precoce do $\mathrm{CM}^{(24)}$, identificando um número significativo de lesões in situ, o que resulta em melhor prognóstico e maiores chances de cura $^{(3)}$, entretanto, a realização do ECM é adjuvante nesse processo, garantindo maior sensibilidade ao rastreamento e detecção precoce.

A realização do ECM demanda tempo reduzido de dedicação por parte dos profissionais, bem como oferece menor custo quando comparado à realização do exame mamográfico ${ }^{(25)}$. Vale ressaltar que a implantação do rastreamento mamográfico em todo o território brasileiro não é uma realidade, devido à falta de infraestrutura e condições econômicas favoráveis para a realização da mamografia e o rastreamento de mulheres com lesões não palpáveis, mesmo diante de avanços reconhecidos nas políticas e programas voltados a ampliar o acesso à mamografia ${ }^{(3,26)}$.

Embora a mamografia seja o exame mais indicado para a detecção precoce do $\mathrm{CM}$ - devido ao seu alto grau de sensibilidade, diagnosticando a doença em estágios iniciais ${ }^{(1)}$-, apenas metade dos médicos e a minoria dos enfermeiros, participantes deste estudo, consideraram a mamografia como o exame mais indicado para a detecção precoce da doença. Esses dados são compatíveis aos encontrados em outro estudo, no qual se observou que o percentual de médicos que citaram a mamografia como método mais adequado para detecção precoce do $\mathrm{CM}$ foi superior ao percentual de enfermeiros ${ }^{(16)}$.

Um estudo realizado na Índia verificou que é possível detectar 8,1 casos a cada mil mulheres em estágio de CM inicial e mais que o dobro, 21,7 casos por mil mulheres, em estágios de CM mais avançados, por meio do ECM. Observou-se, ainda, que houve uma alta frequência de CM em estágios iniciais que não foi detectado por intermédio desse exame ${ }^{(27)}$. Tais resultados demonstram que o ECM não pode ser considerado o método mais eficaz para a detecção precoce do CM, e sua recomendação vem sendo revisada por organizações como a American Cancer Society e pelo 
próprio Ministério da Saúde brasileiro, especialmente pelo alto potencial de resultados falso-positivos ${ }^{(13)}$.

Apesar dos médicos constituírem-se maioria em relação à quantidade de enfermeiros que relacionam a mamografia como exame mais indicado para a detecção precoce, tratase ainda de um número pequeno de profissionais que detêm o conhecimento adequado sobre o assunto, uma vez que o $\mathrm{CM}$ é o câncer com maior índice de mortalidade entre as mulheres $^{(1)}$, e a maioria dos profissionais incluídos neste estudo relatou ter recebido capacitação sobre o tema.

O desconhecimento quanto ao exame é preocupante quando se analisam as duas categorias profissionais que compuseram o estudo, e é ainda maior quando se comparam as repostas entre os médicos e enfermeiros. Em estudo realizado com diversos profissionais da saúde na Nigéria sobre o CM, os médicos demonstraram maior conhecimento sobre a triagem para a realização da mamografia, quando comparados aos enfermeiros e demais categorias ${ }^{(28)}$.

A Política Nacional de Atenção Básica ${ }^{(11)}$ define as atribuições dos profissionais médicos e enfermeiros no âmbito da ESF para o cuidado à família. No caso da mulher em risco ou que desenvolveu o $\mathrm{CM}$, esses profissionais devem apoiar-se nos documentos instrutivos publicados pelo Ministério da Saúde ${ }^{(10,13,20)}$.

A falta de conhecimento ou mesmo o baixo nível deste por parte dos profissionais que atuam no primeiro contato da mulher com o serviço de saúde (APS), como observado neste estudo, influencia a tomada de decisão sobre as condutas adequadas para cada situação. A indicação de exames não adequados retarda o momento do diagnóstico, o que impacta diretamente na oportunidade de cura e aumento da sobrevida da mulher acometida por $\mathrm{CM}^{(3,18)}$.

O ECM é reconhecido por sua relevância como primeiro método de avaliação diagnóstica de lesões mamárias palpáveis, sendo também recomendado em diversos países como método de rastreamento. No Brasil, o ECM esteve recomendado como estratégia de rastreamento para o $\mathrm{CM}$, devendo ser realizado anualmente em mulheres com idade superior a 40 anos e compondo o atendimento integral à saúde da mulher sendo indicado em todas as consultas clínicas, independentemente da idade ${ }^{(20)}$. Entre os profissionais médicos e enfermeiros participantes deste estudo, quase a totalidade declarou não realizar o ECM em todas as consultas.

Em um estudo realizado em Manchester, em 2011, verificou-se que $1 \mathrm{em}$ cada 2 mil mulheres recebeu o diagnóstico de CM após ser avaliada pelo $\operatorname{ECM}(0,5 /$ 1000); em contrapartida, entre as mulheres avaliadas pela mamografia, 1 em cada 193 foi diagnosticada com CM (5,2/ 1000). Dessa forma, podemos constatar que a mamografia possui um grau de sensibilidade mais apurado que o ECM, no entanto, desses resultados, $5,5 \%$ dos casos tiveram falso-negativo nos exames de mamografia e tiveram o $\mathrm{CM}$ detectado em $\mathrm{ECM}^{(25)}$. Assim, é importante salientar que o ECM não apresenta evidências de que pode ser utilizado de forma isolada para a detecção precoce do $\mathrm{CM}^{(12,25)}$. Os dois exames são complementares e de grande importância, especialmente em países como o Brasil, onde a mamografia não é de fácil acesso a todas as mulheres, persistindo a desigualdade nas oportunidades de diagnóstico precoce da doença ${ }^{(25,29)}$.

Vale ressaltar que o grau de sensibilidade do ECM dependerá em grande parte da experiência do profissional, o que garantirá maior segurança na detecção do $\mathrm{CM}^{(30)}$. Embora quase todos os médicos e enfermeiros tenham referido que se sentem capacitados para a realização do ECM, a maioria dos profissionais das duas categorias relatou não oferecer esse exame nas consultas de rotina. Os resultados demonstram que um número expressivo de enfermeiros executa o ECM apenas em mulheres que realizam o exame de Papanicolau, e menos da metade realiza o exame diante de queixas mamárias, como nódulos, dor, descarga capilar e outros sintomas. Resultados diferentes foram identificados entre os médicos, em que mais da metade mencionaram a prática do ECM em mulheres sintomáticas, e uma minoria informou a oferta do exame em mulheres em consulta para realização do Papanicolau. A priorização do ECM apenas em mulheres que buscam o sistema de saúde apresentando sinais e sintomas demonstra uma realidade preocupante, tendo em vista que a fase sintomática do CM está relacionada com estágios mais avançados de progressão da doença ${ }^{(31)}$.

Outra estratégia investigada neste estudo para detecção precoce do $\mathrm{CM}$ diz respeito ao $\mathrm{AEM}$, que embora não seja recomendado como método de detecção do $\mathrm{CM}$, é incentivado para educar as mulheres sobre a saúde geral de mama, encorajando-as a identificar alterações e procurar um profissional de saúde para realizar a avaliação adequada. Sobre a prática do AEM, embora tenha sido recomendado nos últimos 70 anos, estudos não identificaram diferença no impacto sobre os índices de mortalidade, ocasionando ainda o aumento do número de biópsias de lesões benignas e falsa sensação de segurança ${ }^{(1,29,32)}$. No entanto, constatouse em outros estudos que mulheres que realizavam o AEM tiveram menos riscos de serem diagnosticadas com o estágio mais avançado do $\mathrm{CM}^{(29,32)}$. Isso se deve principalmente pelo maior conhecimento do próprio corpo, que a mulher aprimora ao realizar o $\mathrm{AEM}^{(5)}$. Neste estudo, identificouse divergência entre a prática profissional dos enfermeiros quando comparada ao que é preconizado na atualidade para o rastreamento do $\mathrm{CM}$, pois a maioria dos enfermeiros entrevistados considerou o AEM como o mais indicado 
para a detecção precoce da doença. Em contrapartida, os médicos entrevistados consideraram a mamografia o exame mais indicado.

Tendo em vista a elevação das taxas de incidência e mortalidade entre mulheres em decorrência do câncer mamário $^{(1)}$, são necessárias propostas de qualificação e atualizações permanentes direcionadas aos profissionais da APS, que efetivamente repercutam em práticas condizentes com as melhores evidências disponíveis para atenção à saúde das mulheres.

A ampliação das ações com vistas à promoção da saúde e de práticas efetivas de rastreamento, detecção precoce e tratamento do CM deve ser perseguida pelo conjunto de atores (profissionais, gestores e população) envolvidos no enfrentamento a essa doença, considerando-se sua magnitude como problema de saúde pública.

\section{CONCLUSÃO}

Os resultados deste estudo mostraram diferenças significativas tanto no conhecimento quanto na prática referentes às ações para a detecção precoce do CM na ESF. Os médicos apresentaram maior conhecimento sobre a mamografia como método mais adequado para detecção em estágio iniciais do CM quando comparados aos enfermeiros. No que concerne à prática, o ECM é mais realizado pelos médicos em mulheres que apresentam sinais e sintomas mamários já instalados, enquanto os enfermeiros relatam maior realização desse exame nas ocasiões de coleta do Papanicolau.

\section{AGRADECIMENTOS}

Agradecemos ao Professor Albert Schiavetto de Souza, à Secretaria Municipal de Saúde de Campo Grande e ao Programa de Iniciação Científica UFMS/CNPQ.

\section{REFERÊNCIAS}

1. Instituto Nacional de Câncer José Alencar Gomes da Silva. Estimativa 2016: incidência de câncer no Brasil [Internet]. Rio de Janeiro: INCA; 2015 [acesso em 2016 Maio 10]. Disponível em: http://www.inca.gov. br/estimativa/2016/estimativa-2016-v11.pdf

2. Lauter DS, Berlezi EM, Rosanelli CLSP, Loro MM, Kolankiewicz ACB. Câncer de mama: estudo caso controle no Sul do Brasil. Rev Ciência Saúde. 2014;7(1):19-26.

3. Rodrigues JD, Cruz MS, Paixão AN. Uma análise da prevenção do câncer de mama no Brasil. Ciênc Saúde Coletiva [Internet]. 2015 [acesso em 2016
Maio 10];20(10):3163-76. Disponível em: http://www. scielo.br/scielo.php?script=sci_arttext\&pid $=\mathrm{S} 1413$ $-81232015001003163 \& \operatorname{lng}=\mathrm{pt}$

4. Ministério da Saúde (BR), Secretaria de Atenção à Saúde, Departamento de Atenção Básica. Rastreamento [Internet]. Brasília: Ministério da Saúde; 2010. (Série A. Normas e Manuais Técnicos) (Cadernos de Atenção Primária, n. 29) [acesso em 2016 Maio 10]. Disponível em: $\quad$ http://bvsms.saude.gov.br/bvs/publicacoes/ caderno_atencao_primaria_29_rastreamento.pdf

5. Yilmaz D, Bebis $\mathrm{H}$, Ortabag $\mathrm{T}$. Determining the awareness of and compliance with breast cancer screening among Turkish residential women. Asian Pac J Cancer Prev [Internet]. 2013 [acesso em 2016 Maio 10];14:3281-8. Disponível em: http://www. apocpcontrol.org/paper_file/issue_abs/Volume14_ No5/3281-88\%2011.3\%20Demet\%20Yilmaz.pdf

6. Tucunduva LTCM, Sá VHLC, Koshimura ET, Prudente FVB, Santos AF, Samano EST, et al. Estudo da atitude e do conhecimento dos Médicos não Oncologistas em relação às medidas de prevenção e rastreamento do Câncer. Rev Assoc Med Bras. 2004;50(3):257-62.

7. Barreto ASB, Mendes MFM, Thuler LCS. Avaliação de uma estratégia para ampliar a adesão ao rastreamento do câncer de mama no Nordeste brasileiro. Rev Bras Ginecol Obstet. 2012;34(2):86-91.

8. Oliveira AM, Pozer MZ, Silva TA, Parreira BDM, Silva SR. Ações extensionistas voltadas para a prevenção e o tratamento do Câncer Ginecológico e de Mama: relato de experiência. Rev Esc Enferm USP. 2012;46(1):2405 .

9. Gonçalves LLC, Travassos GL, de Almeida AM, Almeida AMDN, Gois CFL. Barriers in health care to breast cancer: perception of women. Rev Esc Enferm USP. 2014;48(3):394-400.

10. Ministério da Saúde (BR), Secretaria de Atenção à Saúde, Departamento de Atenção Básica. Controle dos cânceres do colo do útero e da mama [Internet]. Brasília: Ministério da Saúde; 2013. (Cadernos de Atenção Primária, n. 13) [acesso em 2016 Maio 10]. Disponível em: http://bvsms.saude.gov.br/bvs/ publicacoes/controle_canceres_colo_utero_2013.pdf

11. Ministério da Saúde (BR), Secretaria de Atenção à Saúde, Departamento de Atenção Básica. Política Nacional de Atenção Básica [Internet]. Brasília: Ministério da Saúde; 2012 [acesso em 2016 Maio 10]. Disponível em: http://189.28.128.100/dab/docs/ publicacoes/geral/pnab.pdf 
12. Starfield B. Atenção Primária: equilíbrio entre necessidades de saúde, serviços e tecnologia [Internet]. Brasília: UNESCO; 2002 [acesso em 2016 Maio 10]. Disponível em: http://bvsms.saude.gov.br/bvs/ publicacoes/atencao_primaria_p1.pdf

13. Instituto Nacional de Câncer José Alencar Gomes da Silva. Diretrizes para a detecção precoce do câncer de mama no Brasil [Internet]. Rio de Janeiro: INCA; 2014 [acesso em 2016 Maio 10]. Disponível em: http:// www2.inca.gov.br/wps/wcm/connect/4da965804a441 4659304d3504e7bf539/Diretrizes+Detec\%C3\%A7\% $\mathrm{C} 3 \% \mathrm{~A} 3 \mathrm{o}+$ Precoce $+\mathrm{Ca}+\mathrm{Mama}+2015 . \mathrm{pdf} ? \mathrm{MOD}=\mathrm{AJP}$ ERES\&CACHEID $=4$ da965804a4414659304d3504e7 bf539

14. Shott S. Statistics for health professionals. London: W. B. Saunders Company; 1990.

15. Aráujo GF Junior, Davim RMB. Breast selfexamination practiced by nursing professionals in family health units. J Nurs UFPE . 2012;6(4):759-65.

16. Jácome EM, Silva RM, Gonçalves MLC, Collares PMC, Barbosa IL. Detecção do câncer de mama: conhecimento, atitude e prática dos médicos e enfermeiros da Estratégia Saúde da Família de Mossoró, RN, Brasil. Rev Bras Cancerol [Internet]. 2011 [acesso em 2016 Maio 10]; 57(2):189-98. Disponível em: http://www.inca.gov.br/rbc/n_57/v02/pdf/06_artigo_ deteccao_cancer_mama_conhecimento_atitude pratica_medicos_enfermeiros_estrategia_saude familia_mossoro_RN_brasil.pdf

17. Tesser CD, Garcia AV, Vedrusco AV, Argenta CE. Estratégia saúde da família e análise da realidade social: subsídios para políticas de promoção da saúde e educação permanente. Ciênc Saúde Coletiva [Internet]. 2011 [acesso em 2016 Maio 10];16:4295-306. Disponível em: http://www.scielo.br/pdf/csc/v16n11/ a02v16n11.pdf

18. Lourenço TS, Mauad EC, Vieira RAC. Barreiras no rastreamento do câncer de mama e o papel da enfermagem: revisão integrativa. Rev Bras Enferm [Internet]. 2013 [acesso em 2016 Maio 10 ];66(4):585-91. Disponível em: http://www. scielo.br/scielo.php?script $=$ sci_arttext\&pid $=\mathrm{S} 0034-$ 71672013000400018\&lng=en. $\quad$ http://dx.doi. org/10.1590/S0034-71672013000400018.

19. Gonçalves LLC, Barros ACS, Inagaki ADM, Abid ACF. Avaliação da prática do exame clínico pélvico e de mamas realizados por enfermeiros. Rev Min Enferm [Internet]. 2009 [acesso em 2016 Maio 10];13:244-
8. Disponível em: http://www.reme.org.br/artigo/ detalhes/186

20. Instituto Nacional de Câncer José Alencar Gomes da Silva. Controle do Câncer de Mama: Documento de Consenso [Internet]. Rio de Janeiro: INCA; 2004 [acesso em 2016 Maio 10]. Disponível em: http://www. inca.gov.br/publicacoes/Consensointegra.pdf

21. Moyer VA, U.S. Preventive Services Task Force. Risk assessment, genetic counseling, and genetic testing for BRCA-related cancer in women: U.S. Preventive Services Task Force recommendation statement. Ann Intern Med [Internet]. 2014 [acesso em 2016 Maio 10];160(4):271-81. Disponível em: http://annals.org/ article.aspx?articleid=1791499

22. Nguyen MN, Larocque D, Paquette D, Irace-Cima A. Quebec breast cancer screening program: A study of the perceptions of physicians in Laval, Que. Can Fam Physician. [Internet]. 2009 [acesso em 2016 Maio 10];55(6):614-20. Disponível em: http://www.cfp.ca/ content/55/6/614.full.pdf + html

23. Leite FMC, Amorim MHC, Marques GMT, Vilela APM. Estratégia de saúde da família e o rastreamento do câncer de mama. Espaç Saúde (Online) [Internet]. 2011 [acesso em 2016 Maio 10];12(2):1-9. Disponível em: $\quad$ http://www.uel.br/revistas/uel/index.php/ espacoparasaude/article/view/9170/pdf.

24. Puliti D, Zappa M. Breast cancer screening: are we seeing the benefit? BMC Medicine. 2012; 10:106.

25. Maurice A, Evans G, Affen J, Greenhalgh R, Duffy SW, Howell A. Surveillance of women at increased risk of breast cancer using mammography and clinical breast examination: further evidence of benefit. Int $\mathrm{J}$ Cancer [Internet]. 2011 [acesso em 2016 Maio 10];131:41725. Disponível em: http://onlinelibrary.wiley.com/ doi/10.1002/ijc.26394/epdf

26. Silva GA, Teixeira MTB, Aquino EML, Tomazelli JG, Silva IS. Acesso à detecção precoce do câncer de mama no Sistema Único de Saúde: uma análise a partir dos dados do Sistema de Informações em Saúde. Cad Saúde Pública. 2014;30(7):1537-50.

27. Sankaranarayanan R, Ramadas K, Thara S, Muwonge R, Prabhakar J, Augustine P, et al. Clinical Breast Examination: Preliminary Results from a Cluster Randomized Controlled Trial in India. J Natl Cancer Inst [Internet]. 2011 [acesso em 2016 Maio 10];103(19):1476-80. Disponível em: http://jnci. oxfordjournals.org/content/103/19/1476.long 
28. Akhigbe AO, Omuemu VO. Knowledge, attitudes and practice of breast cancer screening among female health workers in a Nigerian urban city. BMC Cancer [Internet]. 2009 [acesso em 2016 Maio 10];9:203. Disponível em: http://www.ncbi.nlm.nih.gov/pmc/ articles/PMC2709903/pdf/1471-2407-9-203.pdf.

29. Miller AB, Baines CJ. The role of clinical breast examination and breast self-examination. Prev Med. 2011;53(3):118-20.

30. McDonald S, Saslow D, Alciati MH. Performance and Reporting of Clinical Breast Examination: A Review of the Literature. CA Cancer J Clin [Internet]. 2004 [acesso em 2016 Maio 10];54(6):345-61. Disponível em: http://onlinelibrary.wiley.com/doi/10.3322/ canjclin.54.6.345/epdf
31. Thuler LC. Considerações sobre a prevenção do câncer de mama feminino. Rev Bras Cancerol [Internet] 2003 [acesso em 2016 Maio 10];49:227-38. Disponível em: http://www.inca.gov.br/rbc/n_49/v04/pdf/revisao1.pdf

32. Erdem Ö, Toktaş İ. Knowledge, attitudes, and behaviors about breast self-examination and mammography among female primary healthcare workers in Diyarbakır, Turkey. Biomed Res Int. 2016;2016:1-6.

\section{Endereço para correspondência:}

Adriane Pires Batiston

Universidade Federal de Mato Grosso do Sul - UFMS

Rua Robert Spengler, 69

Bairro: Monte Líbano

CEP: 79.004-070 - Campo Grande - MS - Brasil

Email: apbatiston@hotmail.com 